\title{
Association of Premenstrual Syndrome with Body Mass Index, and its Effect on the Quality of Life: A Cross-sectional Study
}

\author{
Mahishale Arati $^{1}$, Joyner C Mesquita ${ }^{2}$
}

\begin{abstract}
Background: Retrospective community surveys estimate that nearly $90 \%$ of women have experienced premenstrual syndrome (PMS) at least once. Premenstrual syndrome is more prevalent among younger women. It also affects the quality of life (QOL) negatively to such a degree that work and lifestyle may get affected.

Objective: To find the association between PMS and body mass index (BMI) and its impact on the QOL.

Materials and methods: A total of 207 females in the 18-25 years age group were screened using Moos menstrual distress questionnaire (MMDQ) for PMS, of which 57 volunteers were diagnosed with PMS. Baseline characteristics were recorded, BMI was calculated, and the QOL of all subjects was measured with the SF-36 questionnaire.

Outcome measures: Body mass index, MMDQ, and SF-36 questionnaire were the outcome measures.

Results: The results showed a significant association between PMS and physical component summary $(\mathrm{PCS} ; r$ value $=-0.4228, p$ value $=0.0011)$ also between PMS and mental component summary (MCS; $r$ value $=-0.4326, p$ value $=0.0008$ ). However, the correlation between PMS and BMI was found to be insignificant.

Conclusion: Premenstrual syndrome affects the QOL negatively; however, no association with BMI was observed.

Keywords: Body mass index, Moos menstrual distress questionnaire, Premenstrual syndrome, Quality of life, SF-36 questionnaire.

Journal of South Asian Federation of Obstetrics and Gynaecology (2019): 10.5005/jp-journals-10006-1746
\end{abstract}

\section{INTRODUCTION}

Premenstrual syndrome (PMS) begins usually 10-14 days earlier to the onset of menstrual period and becomes increasingly worse until the onset of menstruation or several days after the onset. Premenstrual syndrome is defined as the cyclical occurrence of various signs and symptoms beginning near or after ovulation and resolving soon after the commencement of menses. It is the appearance of large collection of symptoms, occurring to such a degree that it affects lifestyle and work quality, and menstruation is followed by a symptom free period. ${ }^{1}$

The symptoms experienced usually include breast tenderness and swelling, weight gain, abdominal bloating, fatigue, depression, and irritability. Headache, constipation, acne, rhinitis, and edema may also occur, as well as more uncommon symptoms occur like paresthesia, sleep disorders, and wide mood swings. Other related symptoms may include poor concentration, sensitivity to noise, and decreased motor skills. ${ }^{1}$

Retrospective community surveys estimate that nearly $90 \%$ of women have experienced PMS at least once as defined by International Classification of Diseases criteria 10. Based on epidemiological surveys conducted, it has been estimated that $75 \%$ of women in their reproductive age experience symptoms attributed to the premenstrual phase of the menstrual cycle. The menstrual cycle has been associated with more than 160 symptoms, ranging from fluid retention and body aches to migraine, fatigue, and headaches, from mood swings to instability. However 3-8\% reproductive age women reported more severe premenstrual symptoms like tension, irritability, dysphoria, and lability of mood, which seriously hampered with their relationships and lifestyle.
${ }^{1}$ Department of OBG Physiotherapy, KLEU Institute of Physiotherapy, J.N. Medical College Campus, Belgaum, Karnataka, India

${ }^{2}$ KLEU Institute of Physiotherapy, J.N. Medical College Campus, Belgaum, Karnataka, India

Corresponding Author: Joyner C Mesquita, KLEU Institute of Physiotherapy, J.N. Medical College Campus, Belgaum, Karnataka, India, Phone: +91 8880303275, e-mail: joynercrystaline@gmail.com

How to cite this article: Arati $M$, Mesquita JC. Association of Premenstrual Syndrome with Body Mass Index, and its Effect on the Quality of Life: A Cross-sectional Study. J South Asian Feder Obst Gynae 2019;11(6):371-374.

Source of support: Nil

Conflict of interest: None

Women's functioning at home, work, and in social situations can be affected substantially every month without relief from these symptoms over the years. This was further named as premenstrual dysphoric disorder (PMDD). ${ }^{2}$

Studies have suggested that data exist providing evidence that obesity and PMS are strongly associated. Hence, PMS management strategies should also consider obesity along with factors like high stress and smoking. ${ }^{3}$ Being overweight $(15 \%$ above the standard weight for height) was associated independently with the probability of longer cycles. Moderate intensity exercise minimally amplified the odds of a long cycle while dieting, tended to shorten expected length. ${ }^{4}$

As per studies, a high rate of PMS in college students affects their life quality adversely. ${ }^{5}$ Premenstrual syndrome (moderate to severe) has negative effect on the health-related quality of life $(\mathrm{QOL}){ }^{6}{ }^{6}$ social 
activities, hobbies, and relationships with others. ${ }^{7}$ However, PMDD places a substantial burden on both mental and physical aspects of health-related QOL. ${ }^{8}$ Adolescents suffering from premenstrual disorders have a poor health-related QOL, and to improve it, appropriate support should be provided especially for those suffering from more severe premenstrual disorders. ${ }^{9}$ Women do not recognize the severity of their PMS symptoms despite the reported difficulties linked with constant social and occupational interference. They are also hesitant to seek aid for treatable PMS symptoms because of attitudinal barriers irrespective of the severity of their symptoms. ${ }^{10}$

A study conducted on overweight women with PMS to evaluate changes in nutrient intake during the menstrual cycle concluded that women with PMS had significantly greater number of "eating episodes" premenstrually. ${ }^{11}$ To the best of our knowledge and literature search association of body mass index (BMI) as well as the QOL with PMS has not been studied, and hence, this study aimed to find the relationship of PMS with BMI and its effect on the QOL.

\section{Materials and Methods}

\section{Source of Data}

Primary data were collected from females with complain of PMS from various educational institutes of Belgaum District, Karnataka.

\section{Study Design}

This study was designed as a cross-sectional study to discover the association between PMS with BMI, and its effect on QOL.

\section{Sampling Design and Sampling Allocation}

Convenience sampling (institutes and students were informed 3 days prior to screening).

\section{Procedure}

- The study was approved by the Institutional Ethical Committee. Two hundred and seven females in the age bracket of 18-25 years were screened using the Moos menstrual distress questionnaire (MMDQ) for PMS.

- Subjects were included based on the fulfillment of the inclusion and exclusion criteria. The inclusion criteria were (1) age between 18 years and 25 years, (2) females with regular menses in last 6 months, (3) unmarried females, (4) subjects with or without medication for PMS, and (5) willingness to participate in the study.

- The exclusion criteria included (1) girls with irregular menstrual cycle in last 6 months, (2) secondary dysmenorrhea, (3) back pain due to neurological origin, and (4) diagnosed with polycystic ovarian disease and known case of other medical disorder.

- An informed consent was signed by all participants that declared their voluntary participation in the study. Out of the returned questionnaires, 57 were diagnosed with PMS.

- Demographic details were taken, and baseline data were recorded. Then, the subjects were assessed for their BMI using the measures of height in meters and weight in kilograms. The subjects were then examined for the impact of PMS on the QOL using the SF-36 questionnaire.

\section{Outcome Measures}

- Body mass index - a formula was used to evaluate obesity which is given by weight (kilograms) divided by the square of height (meters) (BMI $\geq 30 \mathrm{~kg} / \mathrm{m}^{2}$ ).
- Moos menstrual distress questionnaire-the questionnaire consists of 47 items, each describing a "symptom that women sometimes experience;" the subjects are requested to rate the remembrance of their experience of the symptom on a 6-pointer scale, ranging from "no experience of the symptom" to "acute or partially disabling."

- SF-36 questionnaire-the questionnaire comprises 8-scaled scores which are a weighted sum of the questions in their section. Each scale is directly converted into a 0-100-point scale on the assumption that every question holds equal weight. The eight sections are physical functioning, vitality, general health perceptions, bodily pain, emotional role functioning, physical role functioning, social role functioning, and mental health. It analyzes individual patient's health status, monitoring, and comparing disease burden.

\section{Results}

Independent $t$ test was used to estimate the difference among the groups for each outcome. Karl Pearson's correlation test and regression analysis were done to find correlation between the given outcomes. The significance level was set at $p<0.05$. Mean values of baseline characteristics and outcome measures are given in Table 1. Mean age was $20.3 \pm 2.4$ years, and the mean value of BMI measured was $21.6 \pm 3.3 \mathrm{~kg} / \mathrm{m}^{2}$. Mean value of MMDQ was $37.88 \pm 12.8$. Mean physical component summary (PCS) was $43.4 \pm$ 7.4, and mean mental component summary (MCS) was $44 \pm 8.8$.

A correlation analysis was done using Karl Pearson's correlation method. There was a positive association linking PMS and BMI ( $r$ valve $=0.1215, p$ value $=0.3679)$; however, the correlation was not statistically significant. The results displayed a significant relationship between PMS and PCS ( $r$ value $=-0.4228, p$ value $=$ 0.0011 ) and MCS ( $r$ value $=-0.4326, p$ value $=0.0008$ )

A regression analysis was done to evaluate the relationship among variables where PMS was the independent variable and $\mathrm{BMI}$ and QOL were dependent variables. Regression of BMI and PMS scores by MMDQ displayed a linear regression wherein with an increase in BMI, MMDQ scores also increased (refer Fig. 1).

A regression analysis of SF-36 questionnaire-MCS (\%) scores and PCS (\%) with MMDQ (\%) scores-was done. The results displayed a linear regression statistically significant which stated that with an increase in MMDQ scores, there was a decrease in the MCS (\%) scores and PCS scores (refer Figs 2 and 3).

The results of this study showed that there existed a negative impact of PMS and QOL; however, no association was found between PMS and BMI. The prevalence is estimated to be $59 \%$.

\section{Discussion}

A cross-sectional study was conducted to evaluate the correlation between obesity and PMS by Saba Woldemichael Masho in Virginia.

Table 1: Mean values of baseline characteristics and outcome measures

\begin{tabular}{lc}
\hline Variables & \\
\hline Mean age & $20.38596 \pm 2.469$ \\
Mean BMI $\left(\mathrm{kg} / \mathrm{m}^{2}\right)$ & $21.6254386 \pm 3.375$ \\
Mean MMDQ & $37.8807018 \pm 12.838$ \\
Mean PCS & $43.4070175 \pm 7.476$ \\
Mean MCS & $44.0192982 \pm 8.834$ \\
\hline
\end{tabular}




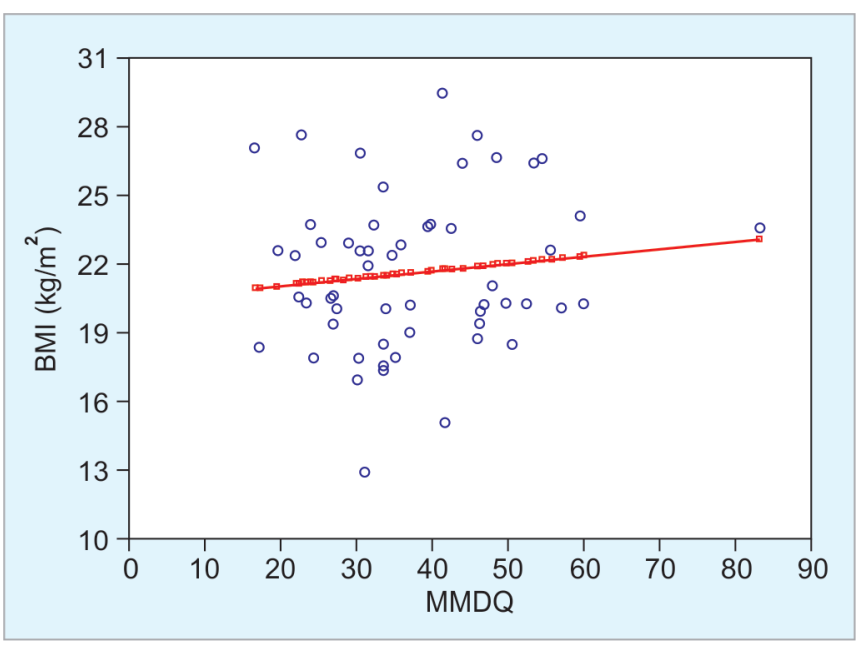

Fig. 1: Regression of body mass index $\left(\mathrm{kg} / \mathrm{m}^{2}\right)$ scores by Moos menstrual distress questionnaire (\%)

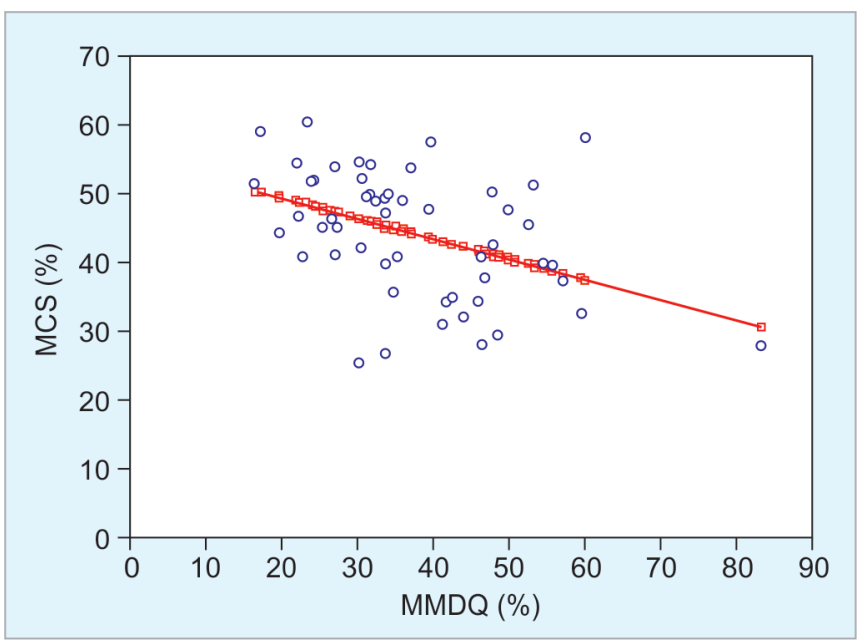

Fig. 3: Regression of mental component summary (\%) scores by Moos menstrual distress questionnaire (\%)

Virginia had a PMS prevalence rate of $10.3 \%$. Obese women $\left(\mathrm{BMI} \geq 30 \mathrm{~kg} / \mathrm{m}^{2}\right)$ presented with nearly a three-fold greater risk for PMS than nonobese women. Premenstrual syndrome was more common among younger women. ${ }^{3}$ In this study, mean BMI of subjects was $\leq 25 \mathrm{~kg} / \mathrm{m}^{2}$ which refers to optimal weight, and hence, no correlation was found between PMS and BMI. However, one inference can be made that PMS exists irrespective of high BMI (Table 2).

A study was done to examine the effect of stress, physical activity, and QOL in women with varying degrees of premenstrual symptomatology by Lustyk et al. which concluded that PMS rate in college students is high, and it negatively affects the life quality. ${ }^{12}$ The current study also found a positive correlation between PMS and QOL. Students had a decreased QOL due to PMS although they were screened at a time where exams were not approaching which could add on to the stress of PMS, and could account for a negative effect on health-related QOL.

A study was conducted to assess the association of the menstrual cycle pattern in 14-17-year-old adolescents with BMI, gynecological age, and historical parameters. The associations between low body weight, stress, weight loss, physical exercise

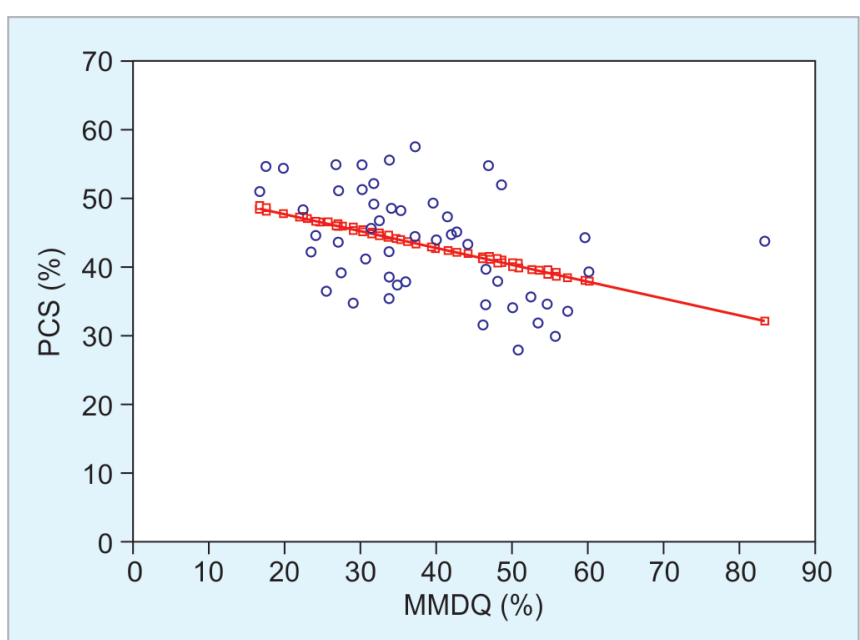

Fig. 2: Regression of physical component summary (\%) scores by Moos menstrual distress questionnaire (\%)

Table 2: Correlation between Moos menstrual distress questionnaire (\%) with body mass index $\left(\mathrm{kg} / \mathrm{m}^{2}\right)$ scores, physical component summary and mental component summary by Karl Pearson's correlation method

\begin{tabular}{lccc}
\hline Variables & rvalue & t value & $p$ value \\
\hline MMDQ (\%) with & 0.1215 & 0.9080 & 0.3679 \\
BMI (kg/m²) & & & \\
MMDQ (\%) with & -0.4228 & -3.4601 & $0.0011^{*}$ \\
PCS (\%) & & & \\
MMDQ (\%) with & -0.4326 & -3.5589 & $0.0008^{*}$ \\
MCS (\%) & & & \\
\hline
\end{tabular}

${ }^{*} p<0.05$

or signs of hyperandrogenism, and menstrual cycle patterns in adolescents are weak when considered on a population basis. The significance of these parameters to explain abnormal menstrual cycle patterns is constrained. ${ }^{13}$ The age of the subjects in this study ranged between 18 years and 22 years. However, other comparisons were not done in this study.

A study was conducted on Explorative Evaluation of the Impact of Severe Premenstrual Disorders on Work Absenteeism and Productivity by Heinemann et al. The study concluded that moderate-to-severe PMS/PMDD seems to be related with work productivity impairment and increased absenteeism, and thus poses an impending economic burden. ${ }^{14}$ In this study also, $33 \%$ of subjects sought leave for their symptoms of PMS which accounted to the loss of attendance and learning impaired sessions.

Astudy was done to assess the relationship between premenstrual symptoms, irregular menstrual cycles, menstrual pain, and psychosocial stress among Japanese college students by Yamamoto et al. The outcome suggests that psychosocial stress is independently linked with premenstrual symptoms and the experience of irregular menstrual cycles among college students, stating that changes in the functional potentiality of women as a consequence of stress are associated with changes in their menstrual function. ${ }^{15}$ The results of this study are consistent with the findings of the above study where PMS had a negative impact on QOL. However, we have excluded subjects with irregular menstrual cycles, and data were collected during cycles which fell during non-examination period.

Premenstrual syndrome adversely affected the learning process and physical and mental well-being in subjects and preventive and 
curative strategies were adopted for management of PMS; however, it was not taken as a part of the study.

There existed a fear of symptoms of PMS which made the girls apprehensive every month prior to the beginning of menses. Hence, it adds to the poor performance and lifestyle not only during the period of menses but also week prior to menses.

\section{References}

1. Rebecca Stephensons G. Obstretics and gynaecological care in physical therapy. 2nd ed., Califonia: Slack incorporated; 2000.

2. Tabassum S, Afridi B, Aman Z, et al. Premenstrual Syndrome: Frequency and Severity in young college girls: observational study. J Pak Med Assoc 2005;55(12):546-549.

3. Masho SW, Adera T, South-Paul J. Obesity as a risk factor for premenstrual syndrome: a cross-sectional study. J Psychosom Obstet Gynaecol 2005;26(1):33-39. DOI: 10.1080/01443610400023049.

4. Harlow SD, Matanoski GM. The association between weight, physical activity, and stress and variation in the length of the menstrual cycle: a cross-sectional study. Am J Epidemiol 1991;133(1):38-49. DOI: 10.1093/ oxfordjournals.aje.a115800.

5. Pinar G, Colak M, Oksuz E. Premenstrual syndrome in turkish college students and its effects on life quality: a cross-sectional study. Sexual and Reproductive Healthcare 2011;2(1):21-27., available from: http:// www.sciencedirect.com/science/article/pii/S1877575610000741.

6. Heinemann LAJ, Do Minh T, Filonenko A, et al. Explorative evaluation of the impact of premenstrual disorder on daily functioning and quality of life. Patient 2010;3(2):125-132.

7. Heinemann LAJ, Minh TD, Filonenko A, et al. Explorative evaluation of the impact of premenstrual disorder on daily functioning and quality of life. Patient 2010;3(2):125-132. DOI: 10.2165/11533750-000000000-00000.
8. Min $\mathrm{Y}$, Gene $\mathrm{W}$, Michael $\mathrm{H}$, et al. Burden of premenstrual dysphoric disorder on health-related quality of life. J Womens Health 2008;17(1):113-121. DOI: 10.1089/jwh.2007.0417.

9. Delara $M$, Ghofranipoul $F$, Azadfallah P, et al. Health related quality of life among adolescents with premenstrual disorders: a cross sectional study. Health Qual Life Outcomes 2012;10:1. DOI: 10.1186/14777525-10-1.

10. Robinson RL, Swindle RW. Premenstrual symptom severity: impact on social functioning and treatment-seeking behaviors. J Womens Health Gend Based Med 2000;9(7):757-768. DOI: 10.1089/15246090050147736.

11. Cross GB, Marley J, Miles $\mathrm{H}$, et al. Changes in nutrient intake during the menstrual cycle of overweight women with premenstrual syndrome. Br J Nutr 2001;85(4):475-482. DOI: 10.1079/BJN2000283.

12. Lustyk MKB, Widman L, Paschane A, et al. Stress, quality of life and physical activity in women with varying degrees of premenstrual symptomatology. Women Health 2004;39(3):35-44. DOI: 10.1300/ J013v39n03_03.

13. van Hooff MHA, Voorhorst FJ, Kaptein MB, et al. The relationship of the menstrual cycle pattern in 14-17 year old adolescents with gynaecological age, body mass index and historical parameters. Hum Reprod 1998;13(8):2252-2260. DOI: 10.1093/humrep/13.8. 2252.

14. Heinemann LAJ, Minh TD, Filonenko A, et al. Explorative evaluation of the impact of severe premenstrual disorders on work absenteeism and productivity. Womens Health Issues 2010;20(1):58-65. DOI: 10.1016/j.whi.2009.09.005.

15. Yamamoto K, Okazaki A, Sakamoto Y, et al. The relationship between premenstrual symptoms, menstrual pain, irregular menstrual cycles, and psychosocial stress among japanese college students. J Physiol Anthropol 2009;28(3):129-136. DOI: 10.2114/jpa2.28.129. 